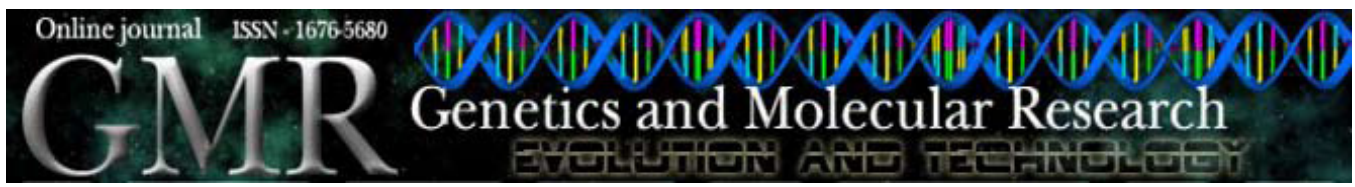

\title{
Genetic diversity of Capsicum chinensis (Solanaceae) accessions based on molecular markers and morphological and agronomic traits
}

\author{
F.L. Finger ${ }^{1}$, S.D. Lannes ${ }^{1}$, A.R. Schuelter ${ }^{2}$, J. Doege ${ }^{2}$, A.P. Comerlato ${ }^{2}$, \\ L.S.A. Gonçalves ${ }^{3}$, F.R.A. Ferreira ${ }^{4}$, L.R. Clovis ${ }^{4}$ and C.A. Scapim ${ }^{4}$ \\ ${ }^{1}$ Departamento de Fitotecnia, Universidade Federal de Viçosa, Viçosa, MG, Brasil \\ ${ }^{2}$ Laboratorio de Biotecnologia, Universidade Paranaense, \\ Campus Toledo, Toledo, PR, Brasil \\ ${ }^{3}$ Laboratorio de Genética e Melhoramento de Plantas, \\ Universidade Estadual do Norte Fluminense Darcy Ribeiro, \\ Campos dos Goytacazes, RJ, Brasil \\ ${ }^{4}$ Departamento de Agronomia, \\ Universidade Estadual de Maringá, Maringá, PR, Brasil
}

Corresponding author: F.L. Finger

E-mail: ffinger@ufv.br

Genet. Mol. Res. 9 (3): 1852-1864 (2010)

Received May 23, 2010

Accepted June 23, 2010

Published September 21, 2010

DOI 10.4238/vol9-3gmr891

\begin{abstract}
We estimated the genetic diversity of 49 accessions of the hot pepper species Capsicum chinensis through analyses of 12 physicochemical traits of the fruit, eight multi-categorical variables, and with 32 RAPD primers. Data from the physicochemical traits were submitted to analysis of variance to estimate the genetic parameters, and their means were clustered by the Scott-Knott test. The matrices from the individual and combined distance
\end{abstract}


were estimated by multivariate analyses before applying Tocher's optimization method. All physicochemical traits were examined for genetic variability by analysis of variance. The responses of these traits showed more contribution from genetic than from environmental factors, except the percentage of dry biomass, content of soluble solids and vitamin $\mathrm{C}$ level. Total capsaicin had the greatest genetic divergence. Nine clusters were formed from the quantitative data based on the generalized distance of Mahalanobis, using Tocher's method; four were formed from the multi-categorical data using the Cole-Rodgers coefficient, and eight were formed from the molecular data using the Nei and Li coefficient. The accessions were distributed into 14 groups using Tocher's method, and no significant correlation between pungency and origin was detected. Uni- and multivariate analyses permitted the identification of marked genetic diversity and fruit attributes capable of being improved through breeding programs.

Key words: Physicochemical traits; Multi-categorical variables; Multivariate analysis; Capsicum chinensis

\section{INTRODUCTION}

The Capsicum genus contains numerous species of sweet and hot peppers (Reifschneider, 2000). The most common are C. annuum L., C. baccatum L., C. chinense Jacq., C. frutescens L., and C. pubescens R. \& P., and horticulturists have cultivated these species worldwide for long time (Casali and Couto, 1984; Moscone et al., 2007). In Brazil, the most indigenous is C. chinensis because this species has a large genetic diversity in the upper Amazon, and it is well adapted to the diverse environmental conditions around the country (Lannes et al., 2007).

Pungency and flavor are fruit attributes of C. chinensis (Bosland, 1993) because of capsaicin and dihydrocapsaicin, which are components of an alkaloid complex responsible for $90 \%$ of the intense organoleptic sensation of heat (Kawada et al., 1985). These fruits are also sources of vitamins $A$, complex $B_{1}$ and $B_{2}$, and minerals such as dietary calcium, iron and phosphorus (Bosland, 1992). The content of vitamin $\mathrm{C}$ in the Capsicum fruit is higher than in Citrus. Furthermore, this species has enormous potential as bedding plants in the ornamental agri-industry because the $C$. chinensis fruit has large variability in size, shape and color (Stommel and Bosland, 2006; Segatto, 2007).

Mature fruit are very important in the agri-industry of dyes, and in numerous processes of the food industry of condiments. They are components of numerous dishes, and may be eaten raw (Souza and Casali, 1984; Bosland, 1993). The agri-industry of dehydrated peppers requires fruit with high dry matter and total soluble solids, thick pericarp and intense color, which are the foremost components for optimizing industrial yields (Casali and Stringheta, 1984). In the raw form, consumers prefer the large and conical fruit that show intense color, brilliant cuticle, smooth pericarp, and firm pulp (Casali et al., 1984). The 
development of new cultivars may expand the Capsicum acreage to supply the ornamental market with bedding plants and the pharmaceutical agri-industry with medicinal plants. However, the success of breeding programs depends on genetic variability for selecting the genotypes with the best commercial characteristics.

The Horticulture Gene Bank of the Universidade Federal de Viçosa (BGH-UFV) has about 100 accessions of $C$. chinensis, in which Teixeira (1996) was the first to detect genetic diversity for fruit size, morphology and color. Next, Lannes et al. (2007) detected large genetic variability for capsaicinoid production. Finally, Pereira (2007) and Schuelter et al. (2010) reported the importance of the genetic factors to determine the fruit characteristics of some accessions. However, more information is still required about the physicochemical traits of these fruit, multi-categorical variables, and random amplified polymorphic DNA (RAPD) molecular markers to find these accessions suitable for breeding programs. In addition, these same data may be used to permit the long-term conservation of $C$. chinensis genotypes in gene banks.

Genetic resources of crop and wild species have been used as the sources of variability for morphological and agronomic traits (Carvalho et al., 2003). Analysis of genetic diversity using quantitative or predictive methods has been used in the composition of populations and lines capable of attending commercial purposes. Multiple data from every accession and their evaluation by predictive analyses are expressed by dissimilarity indices, which represent the diversity of several groups (Cruz and Carneiro, 2003). Recently, this divergence was evaluated by individual analyses of genetic distance and combined analyses (Gonçalves et al., 2008; Kumar et al., 2009). Furthermore, several studies have evaluated the genetic diversity of morphological and agronomic traits (Teixeira, 1996; Oliveira et al., 1999; Rêgo et al., 2003) and molecular markers of Capsicum (Paran et al., 1998; Buso et al., 2003; Toquica et al., 2003).

Thus, the objective of the present study was to document the characteristics of 49 accessions of $C$. chinensis stored in the BGH-UFV using RAPD markers, to estimate the genetic diversity of physicochemical fruit traits, and to evaluate multi-categorical variables through multivariate analyses.

\section{MATERIAL AND METHODS}

\section{Material and crop conditions}

Forty-nine accessions of $C$. chinensis collected in different Brazilian regions and stored in the BGH-UFV (Table 1) were selected based on their fruit appearance and plant architecture. Their physicochemical fruit traits and the multi-categorical variables were evaluated in the Department of Plant Production, Viçosa, Minas Gerais State, Brazil. The RAPD analyses, otherwise, were carried out in the Biotechnology Laboratory of the Universidade Paranaense, Toledo, Paraná State, Brazil.

Pepper seeds germinated on Styrofoam trays filled with organic growing medium. The 45-day-old bedding plants were transplanted to the field in a completely randomized block design with three replications. Every replication had the plants spaced $0.5 \mathrm{~m}$ within rows and $1.0 \mathrm{~m}$ between rows, where the morphological and agronomic traits were evaluated from the inner four in six plants. 
Table 1. Origin of 49 accessions of Capsicum chinensis stored in the BGH-UFV and their characteristics evaluated by qualitative multi-categorical traits.

\begin{tabular}{|c|c|c|c|c|c|c|c|c|c|}
\hline \multirow[t]{2}{*}{ Accession } & \multirow[t]{2}{*}{ Origin } & \multicolumn{8}{|c|}{ Multicategorical traits } \\
\hline & & $\mathrm{GH}$ & SP & $\mathrm{SC}$ & $\mathrm{CC}$ & FS & IFC & MFC & SFT \\
\hline BGH 1694-5 & Cuiabá, MT & 5 & 1 & 1 & 1 & 6 & 1 & 3 & 7 \\
\hline BGH 1694-6 & Cuiabá, MT & 5 & 3 & 1 & 1 & 6 & 1 & 4 & 7 \\
\hline BGH 1694-7 & Cuiabá, MT & 5 & 5 & 1 & 1 & 6 & 1 & 4 & 7 \\
\hline BGH 1714-9 & Pindaré-Mirim, MA & 7 & 3 & 1 & 1 & 4 & 1 & 3 & 3 \\
\hline BGH 1714-11 & Pindaré-Mirim, MA & 5 & 3 & 1 & 1 & 5 & 1 & 4 & 3 \\
\hline BGH 1716-14 & Pindaré-Mirim, MA & 3 & 3 & 1 & 1 & 1 & 1 & 3 & 3 \\
\hline BGH 1716-16 & Pindaré-Mirim, MA & 7 & 3 & 1 & 1 & 4 & 2 & 2 & 3 \\
\hline BGH 1716-17 & Pindaré-Mirim, MA & 5 & 3 & 1 & 1 & 1 & 1 & 2 & 3 \\
\hline BGH 1716-18 & Pindaré-Mirim, MA & 5 & 3 & 1 & 1 & 1 & 1 & 2 & 3 \\
\hline BGH 1716-19 & Pindaré-Mirim, MA & 5 & 3 & 1 & 1 & 1 & 1 & 3 & 3 \\
\hline BGH 1723-22 & Santarém, AM & 7 & 5 & 2 & 1 & 5 & 1 & 3 & 3 \\
\hline BGH 1724-23 & Santarém, AM & 7 & 3 & 1 & 1 & 1 & 1 & 4 & 3 \\
\hline BGH 1747-26 & Ituiutaba, MG & 7 & 3 & 1 & 1 & 3 & 1 & 4 & 3 \\
\hline BGH 1747-27 & Ituiutaba, MG & 5 & 5 & 1 & 1 & 4 & 1 & 4 & 3 \\
\hline BGH 4199-30 & Belém, PA & 5 & 7 & 1 & 1 & 5 & 1 & 4 & 3 \\
\hline BGH 4201-32 & Belém, PA & 7 & 3 & 2 & 1 & 4 & 1 & 3 & 3 \\
\hline BGH 4213-34 & Tefé, AM & 7 & 5 & 1 & 1 & 1 & 5 & 3 & 7 \\
\hline BGH 4223-39 & Campinas, SP & 5 & 5 & 2 & 1 & 1 & 1 & 4 & 3 \\
\hline BGH 4285-40 & Rondonópolis, MT & 7 & 7 & 1 & 1 & 4 & 1 & 4 & 3 \\
\hline BGH 4289-44 & Rondonópolis, MT & 7 & 3 & 1 & 1 & 1 & 1 & 4 & 3 \\
\hline BGH 4289-45 & Rondonópolis, MT & 5 & 3 & 1 & 1 & 1 & 1 & 3 & 3 \\
\hline BGH 4355-46 & Pedro Afonso, GO & 7 & 5 & 1 & 1 & 6 & 1 & 3 & 5 \\
\hline BGH 4725-51 & Urucará, AM & 5 & 5 & 1 & 1 & 4 & 1 & 3 & 3 \\
\hline BGH 4731-53 & Autazes-Mirim, AM & 7 & 3 & 1 & 1 & 4 & 1 & 3 & 3 \\
\hline BGH 4733-54 & Manaus, AM & 7 & 5 & 2 & 1 & 1 & 1 & 4 & 3 \\
\hline BGH 4733-55 & Manaus, AM & 5 & 5 & 1 & 1 & 1 & 1 & 4 & 3 \\
\hline BGH 4733-56 & Manaus, AM & 7 & 5 & 1 & 1 & 1 & 1 & 4 & 3 \\
\hline BGH 4744-57 & Canabuoca-Manacapuru, AM & 5 & 3 & 2 & 1 & 5 & 1 & 4 & 3 \\
\hline BGH 4750-59 & Joari-Itacotiara, AM & 7 & 3 & 1 & 1 & 1 & 1 & 4 & 3 \\
\hline BGH 4756-67 & Joari-Itacotiara, AM & 5 & 5 & 1 & 1 & 1 & 1 & 4 & 3 \\
\hline BGH 4756-70 & Joari-Itacotiara, AM & 5 & 3 & 1 & 1 & 1 & 1 & 4 & 3 \\
\hline BGH 4756-71 & Joari-Itacotiara, AM & 7 & 3 & 1 & 1 & 1 & 2 & 4 & 3 \\
\hline BGH 5012-72 & Água Branca, AL & 5 & 5 & 1 & 1 & 1 & 2 & 3 & 3 \\
\hline BGH 5012-76 & Água Branca, AL & 7 & 3 & 1 & 1 & 1 & 2 & 3 & 3 \\
\hline BGH 6009-78 & Belém, PA & 5 & 3 & 1 & 1 & 5 & 1 & 4 & 3 \\
\hline BGH 6228-79 & Brasília, DF & 5 & 3 & 1 & 1 & 1 & 1 & 3 & 3 \\
\hline BGH 6228-82 & Brasília, DF & 7 & 5 & 1 & 1 & 4 & 1 & 4 & 3 \\
\hline BGH 6233-83 & Brasília, DF & 5 & 5 & 1 & 1 & 1 & 1 & 4 & 3 \\
\hline BGH 6233-84 & Brasília, DF & 7 & 3 & 1 & 1 & 4 & 1 & 4 & 3 \\
\hline BGH 6233-85 & Brasília, DF & 5 & 3 & 1 & 1 & 4 & 1 & 4 & 3 \\
\hline BGH 6239-86 & Brasília, DF & 7 & 3 & 1 & 1 & 1 & 1 & 4 & 3 \\
\hline BGH 6344-87 & Dourados, MS & 5 & 3 & 2 & 1 & 2 & 5 & 3 & 5 \\
\hline BGH 6369-90 & Vila Nova-Anamam, AM & 5 & 3 & 1 & 1 & 4 & 1 & 4 & 7 \\
\hline BGH 6371-93 & Vila Nova-Anamam, AM & 5 & 3 & 1 & 2 & 1 & 1 & 4 & 3 \\
\hline BGH 6371-94 & Vila Nova-Anamam, AM & 5 & 3 & 1 & 2 & 1 & 1 & 4 & 3 \\
\hline BGH 6371-95 & Vila Nova-Anamam, AM & 7 & 1 & 2 & 1 & 6 & 1 & 4 & 3 \\
\hline BGH 6378-98 & Boca do Janacanan-Codajas, AM & 5 & 3 & 1 & 1 & 4 & 1 & 4 & 3 \\
\hline BGH 6387-100 & Salvador, BA & 7 & 3 & 2 & 1 & 6 & 1 & 4 & 7 \\
\hline BGH 6515-101 & Viçosa, MG & 7 & 1 & 2 & 1 & 2 & 1 & 3 & 3 \\
\hline
\end{tabular}

$\mathrm{GH}=$ growth habit; $\mathrm{SP}=$ stem pubescence; $\mathrm{SC}=$ stem color; $\mathrm{CC}=$ corolla color; $\mathrm{FS}=$ fruit shape; $\mathrm{IFC}=$ immature fruit color; $\mathrm{MFC}=$ mature fruit color; $\mathrm{SFT}=$ shape of the fruit tip.

\section{Morphological and agronomic traits}

In five mature fruits, fruit length and width, pulp thickness, total fresh biomass, total dry biomass, percentage of dry biomass, content of soluble solids, content of vitamin C, color intensity, capsaicin, and dihydrocapsaicin using the method of Maillard et al. (1997), and the sum of both capsaicinoids were all evaluated in the Post-Harvest Laboratory at the Department of Plant Production in the Universidade Federal de Viçosa. Vitamin C was determined using Tillmans' method 
(Instituto Adolfo Lutz, 1985), and color intensity was evaluated using the ASTA method 20-1.

Twelve plants of every accession were examined following the characteristics: prostrate (3), compact (5), or erect (7) growth habit; absent (1), scarce (3), moderate (5), or abundant (7) stem pubescence; elongated (1), triangular (2), rounded (3), conical (4), campanular (5), or bell (6) fruit shape; pointed (3), blunt-tipped and absent (5) or sunken (7) fruit apex; green (1) or purple (2) stem color; white (1), white-greenish (2) or green (2) corolla; green (1), yellow (2), orange (3), red (4), brown (6), or black (7) immature fruit, and green (1), yellow (2), orange (3), red (4), purple (5), brown (6), or black (7) mature fruit. These eight multi-categorical traits were evaluated using the keys of minimum descriptors available at the International Plant Genetic Resource Institute (IPGRI, 1995).

\section{RAPD molecular markers}

Six-day-old leaf blades were collected from bedding plants to characterize the accessions using the RAPD markers. The protocol for DNA extraction and purification was described by Fulton et al. (1995), and the amplifications were carried out on the Thermo PX2 thermocycler (Williams et al., 1990). The amplified fragments were submitted to horizontal electrophoresis on $1.2 \%$ agarose gel, stained with $2 \mathrm{mg} / \mathrm{L}$ ethidium bromide and revealed by a photo documentation system.

\section{Statistical analyses}

Data of physicochemical traits were submitted to the analysis of variance to determine genetic variability, and the Scott-Knott test (1974) was used to cluster the accessions. Estimates of genetic and environmental parameters were used to evaluate the breeding chances of these traits.

The presence of multicollinearity in the correlation matrix was investigated before the multivariate analyses to eliminate any bias in the estimates of genetic parameters. Afterward, the generalized Mahalanobis distance was used to determine the dissimilarity matrix for the quantitative traits, Cole-Rodgers et al.' coefficient (1997) for the multi-categorical traits, and Nei and Li's coefficient (1979) for the molecular markers. Next, the Mantel test (1967) was applied to determine the association between the genetic distance matrices.

The clusters were formed by optimizing Tocher's method (Rao, 1952) through the different genetic distance matrices; the relative contribution of every trait to genetic divergence was estimated by Singh's method (1981). The analyses of canonical variables to eliminate those physicochemical traits with no influence on Tocher's clustering were suggested by Rêgo et al. (2003). All analyses were carried out using the GENES software (Cruz, 2006a,b).

\section{RESULTS AND DISCUSSION}

All responses from the physicochemical traits (Table 2$)$ were significantly different $(\mathrm{P}<$ 0.01 ) and showed evidence of genetic variability between the genotypes. Data of fruit length and width, pulp thickness, capsaicin, dihydrocapsaicin, total capsaicinoids, soluble solids, and vitamin $\mathrm{C}$ had the coefficients of variation between 5.30 and $20.44 \%$, which accounted for accuracy in the experimental errors. Otherwise, the magnitude of these coefficients from data for fresh and dry biomass and fruit color indicated discrepancy among the accessions. Usually, as these traits are affected by the environment, there are difficulties in controlling them under experimental conditions. 
Genetic diversity of Capsicum chinensis

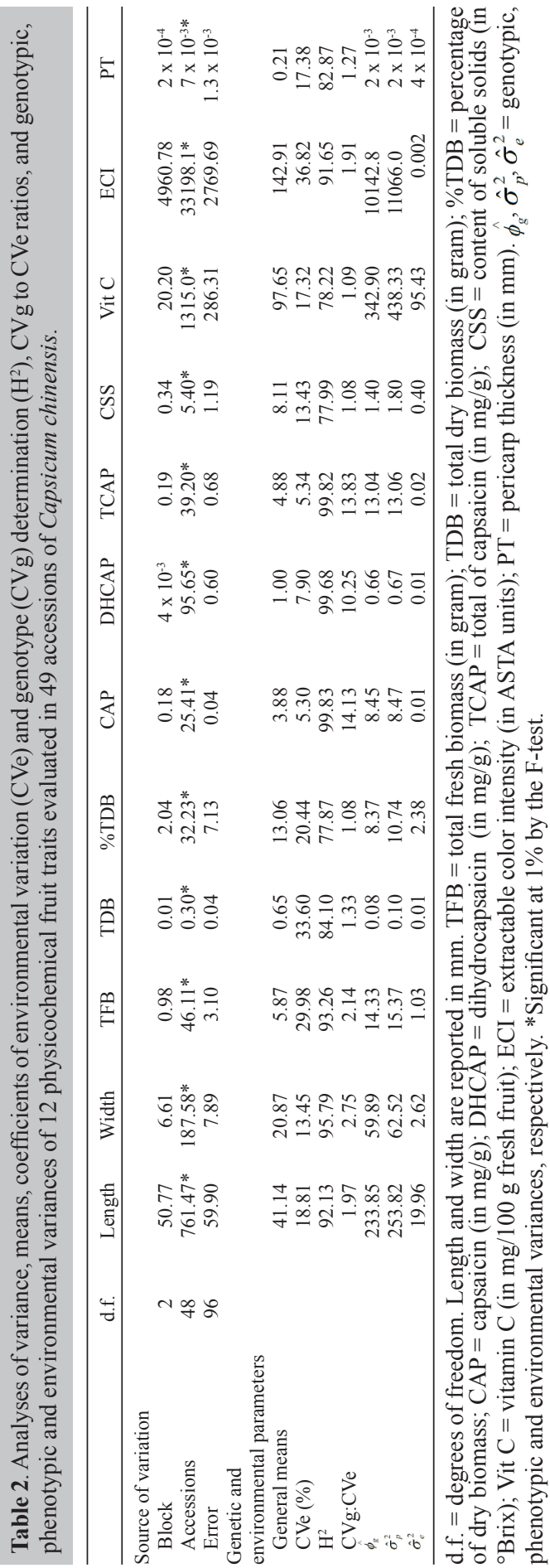


The importance of genetic factors on these morphological and agronomic traits is indicated by the high genotypic coefficients of determination between 77.87 and $99.83 \%$. Therefore, fruit length and width, fresh biomass, capsaicin, dihydrocapsaicin, total capsaicinoids, dry biomass, pericarp thickness, and extractable color can be selected by the mass method, because the genotypic coefficients of determination were above $80 \%$, the ratio $\mathrm{CVg}$ to Cve was higher than one, and there was high genetic variance. Otherwise, breeding methods capable of controlling the environment must be used to select plants with high percentage of dry biomass, total soluble solids and vitamin $\mathrm{C}$, because the coefficient of determination from the genotypes was close to one.

The Scott-Knott test $(\mathrm{P}<0.05)$ allowed the clustering of these accessions into five groups based on the fruit length between 14.15 and $76.22 \mathrm{~mm}$. The accessions BGH 6371-93, BGH 4733-54, BGH 4223-39, and BGH 4285-40 are clustered into group A because of their long fruit, unlike BGH 6515-101 and BGH 4289-44, which are clustered into an opposite group because of their short fruit. Otherwise, all the accessions are clustered into six groups based on fruit width. The cluster A has just BGH 6009-78 with an estimate of $42.89 \mathrm{~mm}$, but the group B contains the accessions BGH 4355-46, BGH 4731-53, BGH 6233-84, and BGH 6371-95. Eighty-seven percent of the accessions, however, have fruit width less than $30 \mathrm{~mm}$. The ratio fruit length to fruit width ranges between 0.68 and 6.61 , and long fruit is the predominant shape. The BGH 6371-94 accession shows the highest ratio.

Pulp thickness ranges from 1.0 to $3.5 \mathrm{~mm}$, and all the accessions were clustered into only three groups in which BGH 6009-78, with the thickest pulp, was clustered together with BGH 4223-39, BGH 6233-85, BGH 6378-98, BGH 6369-90, and BGH 1694-07; all of them have about $3.0 \mathrm{~mm}$ of pulp. Other accessions were clustered into group B $(28.6 \%)$ because of their moderate pulp thickness and C (59.2\%) because of their thinner pulps. The accession BGH 6371-94 had $1.17 \mathrm{~mm}$ thickness and BGH 4733-56 had $1.11 \mathrm{~mm}$ thickness, representing the thinnest fruit pulp (Table 3).

The total biomass is between 0.99 and $19.15 \mathrm{~g}$. In the cluster A, BGH 6009-78 is the only accession with the highest estimate $(\mathrm{P}<0.05)$, and it is followed by the cluster $\mathrm{B}$ with BGH 6233-84, BGH 4223-39, BGH 4731-53, and BGH 6233-85. Ninety percent of the accessions, otherwise, have estimates below $11 \mathrm{~g}$, and they were clustered into C, D and E.

Fresh biomass is predominantly lower among the accessions (Table 3 ). High fruit dehydration accounted for the dry biomass between 0.17 and $1.42 \mathrm{~g}$, and only four clusters were formed using the Scott-Knott test $(\mathrm{P}<0.05)$. BGH 6009-78, BGH 4223-39 and BGH 4731-53 with high fresh biomass together with BGH 6371-93 were clustered into group A. Otherwise, BGH 6233-84 and BGH 6233-85, which were clustered into A for total biomass, were here clustered into B. This fact raises the importance of the water content because of the large variation, between 7.05 and $20.92 \%$, in the percentage of dry biomass in these genotypes. The accessions were distributed into the groups with high (A) and low (B) percentage of dry biomass $(\mathrm{P}<0.05)$. Cluster A with $31 \%$ of the accessions had the minimum dry biomass equal to $14.42 \%$, although BGH $4733-56$ and BGH $4289-44$ had percentages of about $21 \%$ despite the low level of total fresh biomass in these small fruit. Similar responses were reported by Lannes et al. (2007), who found a negative correlation between these traits. Nevertheless, both traits have sufficient genetic variability to participate in the genotype selection to supply the fresh fruit market or the food industry with new cultivars.

The content of total soluble solids ranges between 5.93 and $12.90^{\circ}$ Brix, and all the accessions were clustered into only three groups. BGH 4733-56, with the highest content, was 


\begin{tabular}{|c|c|c|c|c|c|c|c|c|c|c|c|c|}
\hline Accession & Length & Width & TFB & TDB & $\%$ TDB & CSS & Vit C & ECI & $\mathrm{PT}$ & CAP & DHCAP & TCAP \\
\hline BGH 1694-5 & $27.78^{\mathrm{e}}$ & $22.17^{\mathrm{d}}$ & $3.55^{\mathrm{e}}$ & $0.50^{\mathrm{c}}$ & $13.90^{\mathrm{b}}$ & $8.36^{\mathrm{c}}$ & $98.65^{\mathrm{a}}$ & $88.78^{\mathrm{e}}$ & $0.18^{\mathrm{c}}$ & $3.91^{\mathrm{k}}$ & $0.62^{\mathrm{k}}$ & $4.53^{1}$ \\
\hline BGH 1694-6 & $23.43^{\mathrm{e}}$ & $21.27^{\mathrm{d}}$ & $3.18^{\mathrm{e}}$ & $0.41^{\mathrm{d}}$ & $12.91^{\mathrm{b}}$ & $8.49^{\mathrm{c}}$ & $81.41^{\mathrm{b}}$ & $229.08^{c}$ & $0.17^{\mathrm{c}}$ & $3.89^{k}$ & $0.81^{\mathrm{j}}$ & $4.70^{1}$ \\
\hline BGH 1694-7 & $33.40^{\mathrm{d}}$ & $28.55^{\mathrm{c}}$ & $9.65^{\mathrm{c}}$ & $0.98^{b}$ & $10.21^{\mathrm{b}}$ & $6.48^{c}$ & $83.65^{\mathrm{b}}$ & $123.97^{\mathrm{d}}$ & $0.28^{\mathrm{a}}$ & $1.42^{\circ}$ & $0.45^{1}$ & $1.87^{\mathrm{q}}$ \\
\hline BGH 1714-9 & $41.82^{\mathrm{d}}$ & $21.99^{\mathrm{d}}$ & $5.92^{\mathrm{d}}$ & $0.86^{\mathrm{b}}$ & $16.73^{\mathrm{a}}$ & $8.90^{\mathrm{b}}$ & $91.65^{\mathrm{b}}$ & $105.54^{\mathrm{e}}$ & $0.24^{\mathrm{b}}$ & $1.70^{\circ}$ & $0.44^{1}$ & $2.14^{\mathrm{p}}$ \\
\hline BGH 1714-11 & $40.32^{\mathrm{d}}$ & $25.46^{\mathrm{c}}$ & $5.58^{\mathrm{d}}$ & $0.62^{\mathrm{c}}$ & $10.79^{b}$ & $7.20^{\mathrm{c}}$ & $86.42^{\mathrm{b}}$ & $181.92^{\mathrm{d}}$ & $0.22^{\mathrm{b}}$ & $1.89^{\mathrm{n}}$ & $0.47^{1}$ & $2.36^{\mathrm{p}}$ \\
\hline BGH 1716-14 & $36.81^{\mathrm{d}}$ & $19.09^{\mathrm{d}}$ & $4.28^{\mathrm{d}}$ & $0.49^{\mathrm{c}}$ & $11.68^{\mathrm{b}}$ & $8.59^{\mathrm{b}}$ & $80.95^{\mathrm{b}}$ & $56.66^{\mathrm{e}}$ & $0.20^{\mathrm{c}}$ & $0.00^{\mathrm{q}}$ & $0.00^{\mathrm{m}}$ & $0.00^{\mathrm{s}}$ \\
\hline BGH 1716-16 & $37.74^{\mathrm{d}}$ & $20.77^{\mathrm{d}}$ & $6.06^{\mathrm{d}}$ & $0.72^{\mathrm{c}}$ & $12.45^{\mathrm{b}}$ & $9.44^{b}$ & $86.31^{\mathrm{b}}$ & $30.35^{\mathrm{e}}$ & $0.25^{\mathrm{b}}$ & $0.00^{\mathrm{q}}$ & $0.00^{\mathrm{m}}$ & $0.00^{\mathrm{s}}$ \\
\hline BGH 1716-17 & $52.29^{\mathrm{c}}$ & $13.73^{\mathrm{e}}$ & $4.28^{\mathrm{d}}$ & $0.52^{\mathrm{c}}$ & $12.51^{\mathrm{b}}$ & $8.29^{c}$ & $101.31^{\mathrm{a}}$ & $75.25^{\mathrm{e}}$ & $0.20^{\mathrm{c}}$ & $10.32^{\mathrm{a}}$ & $3.30^{\mathrm{a}}$ & $13.62^{\mathrm{a}}$ \\
\hline BGH 1716-18 & $54.69^{c}$ & $17.55^{\mathrm{d}}$ & $5.80^{\mathrm{d}}$ & $0.66^{\mathrm{c}}$ & $11.83^{\mathrm{b}}$ & $8.80^{\mathrm{b}}$ & $106.15^{\mathrm{a}}$ & $56.75^{\mathrm{e}}$ & $0.19^{\mathrm{c}}$ & $5.58^{\mathrm{h}}$ & $1.18^{\mathrm{h}}$ & $6.76^{\mathrm{i}}$ \\
\hline BGH 1716-19 & $62.83^{\mathrm{b}}$ & $14.17^{\mathrm{e}}$ & $5.29^{\mathrm{d}}$ & $0.57^{\mathrm{c}}$ & $11.63^{\mathrm{b}}$ & $8.30^{\mathrm{c}}$ & $100.92^{\mathrm{a}}$ & $60.21^{\mathrm{e}}$ & $0.16^{\mathrm{c}}$ & $1.45^{\circ}$ & $0.40^{1}$ & $1.85^{\mathrm{q}}$ \\
\hline BGH 1723-22 & $29.56^{\mathrm{e}}$ & $22.32^{\mathrm{d}}$ & $4.81^{\mathrm{d}}$ & $0.50^{\mathrm{c}}$ & $10.45^{\mathrm{b}}$ & $7.75^{\mathrm{c}}$ & $99.47^{\mathrm{a}}$ & $130.93^{\mathrm{d}}$ & $0.19^{c}$ & $3.13^{\mathrm{m}}$ & $1.78^{\mathrm{e}}$ & $4.91^{\mathrm{k}}$ \\
\hline BGH 1724-23 & $21.71^{\mathrm{e}}$ & $12.87^{\mathrm{e}}$ & $1.65^{\mathrm{e}}$ & $0.23^{\mathrm{d}}$ & $14.72^{\mathrm{a}}$ & $8.26^{\mathrm{c}}$ & $78.60^{\mathrm{b}}$ & $234.57^{\mathrm{c}}$ & $0.16^{\mathrm{c}}$ & $7.15^{f}$ & $1.35^{\mathrm{g}}$ & $8.50^{\mathrm{f}}$ \\
\hline BGH 1747-26 & $16.57^{\mathrm{e}}$ & $15.27^{\mathrm{e}}$ & $1.38^{\mathrm{e}}$ & $0.25^{\mathrm{d}}$ & $18.27^{\mathrm{a}}$ & $7.64^{\mathrm{c}}$ & $118.94^{\mathrm{a}}$ & $383.74^{\mathrm{b}}$ & $0.19^{c}$ & $2.80^{\mathrm{m}}$ & $0.56^{1}$ & $3.36^{\mathrm{n}}$ \\
\hline BGH $1747-27$ & $37.17^{\mathrm{d}}$ & $19.53^{\mathrm{d}}$ & $4.22^{\mathrm{d}}$ & $0.54^{\mathrm{c}}$ & $13.68^{\mathrm{b}}$ & $8.81^{\mathrm{b}}$ & $97.48^{\mathrm{a}}$ & $273.77^{\mathrm{c}}$ & $0.18^{\mathrm{c}}$ & $3.84^{k}$ & $0.10^{\mathrm{i}}$ & $4.84^{1}$ \\
\hline BGH 4199-30 & $39.47^{\mathrm{d}}$ & $26.71^{\mathrm{c}}$ & $6.96^{\mathrm{d}}$ & $0.68^{c}$ & $10.40^{\mathrm{b}}$ & $7.19^{c}$ & $107.57^{\mathrm{a}}$ & $140.49^{d}$ & $0.24^{\mathrm{b}}$ & $4.04^{\mathrm{k}}$ & $1.00^{\mathrm{i}}$ & $5.04^{k}$ \\
\hline BGH 4201-32 & $48.33^{\mathrm{c}}$ & $17.91^{\mathrm{d}}$ & $5.67^{\mathrm{d}}$ & $0.75^{\mathrm{b}}$ & $12.74^{\mathrm{b}}$ & $9.09^{\mathrm{b}}$ & $113.15^{\mathrm{a}}$ & $38.96^{\mathrm{e}}$ & $0.24^{\mathrm{b}}$ & $0.00^{\mathrm{q}}$ & $0.00^{\mathrm{m}}$ & $0.00^{\mathrm{s}}$ \\
\hline BGH 4213-34 & $25.39^{\mathrm{e}}$ & $18.58^{\mathrm{d}}$ & $2.88^{\mathrm{e}}$ & $0.45^{\mathrm{c}}$ & $16.05^{\mathrm{a}}$ & $7.88^{\mathrm{c}}$ & $112.05^{\mathrm{a}}$ & $111.50^{\mathrm{e}}$ & $0.20^{\mathrm{c}}$ & $4.86^{\mathrm{i}}$ & $1.76^{\mathrm{e}}$ & $6.62^{\mathrm{i}}$ \\
\hline BGH 4223-39 & $70.43^{\mathrm{a}}$ & $22.98^{d}$ & $13.08^{\mathrm{b}}$ & $1.42^{\mathrm{a}}$ & $10.91^{\mathrm{b}}$ & $7.03^{c}$ & $100.07^{\mathrm{a}}$ & $133.86^{d}$ & $0.30^{\mathrm{a}}$ & $0.00^{\mathrm{q}}$ & $0.00^{\mathrm{m}}$ & $0.00^{\mathrm{s}}$ \\
\hline BGH $4285-40$ & $70.27^{\mathrm{a}}$ & $24.81^{\mathrm{c}}$ & $9.50^{\mathrm{c}}$ & $0.95^{\mathrm{b}}$ & $10.34^{\mathrm{b}}$ & $6.59^{\mathrm{c}}$ & $111.66^{\mathrm{a}}$ & $174.51^{\mathrm{d}}$ & $0.20^{\mathrm{c}}$ & $3.99^{\mathrm{k}}$ & $1.07^{\mathrm{i}}$ & $5.06^{\mathrm{k}}$ \\
\hline BGH 4289-44 & $19.41^{\mathrm{e}}$ & $10.89^{\mathrm{f}}$ & & $0.20^{\mathrm{d}}$ & $20.53^{\mathrm{a}}$ & $10.86^{\mathrm{a}}$ & $76.01^{\mathrm{b}}$ & $34.87^{\mathrm{e}}$ & $0.14^{\mathrm{c}}$ & & $3.42^{\mathrm{a}}$ & $8.13^{\mathrm{f}}$ \\
\hline BGH $4289-45$ & $40.13^{\mathrm{d}}$ & 11.4 & & 0. & & & 119. & 80 . & & & & $6.18^{\mathrm{j}}$ \\
\hline BGH 4355-46 & $27.27^{\mathrm{e}}$ & $34.68^{\mathrm{b}}$ & $6.77^{d}$ & $0.64^{\mathrm{c}}$ & $10.56^{\mathrm{b}}$ & $6.75^{\mathrm{c}}$ & $56.17^{\mathrm{c}}$ & $50.70^{\mathrm{e}}$ & $0.17^{\mathrm{c}}$ & $4.94^{\mathrm{i}}$ & & $5.99^{j}$ \\
\hline BGH 4725-51 & $33.92^{\mathrm{d}}$ & $20.07^{\mathrm{d}}$ & $4.24^{\mathrm{d}}$ & $0.55^{\mathrm{c}}$ & $13.66^{\mathrm{b}}$ & $7.46^{\mathrm{c}}$ & $113.40^{\mathrm{a}}$ & $62.82^{\mathrm{e}}$ & $0.25^{\mathrm{b}}$ & $3.69^{k}$ & $1.03^{\mathrm{i}}$ & $4.72^{1}$ \\
\hline BGH 4731-53 & $58.17^{\mathrm{b}}$ & $33.95^{\mathrm{b}}$ & $12.46^{\mathrm{b}}$ & $1.17^{\mathrm{a}}$ & $9.76^{\mathrm{b}}$ & $7.20^{\mathrm{c}}$ & $81.62^{\mathrm{b}}$ & $54.66^{\mathrm{e}}$ & $0.24^{\mathrm{b}}$ & $0.00^{\mathrm{q}}$ & $0.00^{\mathrm{m}}$ & $0.00^{\mathrm{s}}$ \\
\hline BGH 4733-54 & $74.16^{\mathrm{a}}$ & $20.02^{\mathrm{d}}$ & $6.65^{\mathrm{d}}$ & $0.86^{\mathrm{b}}$ & $12.83^{\mathrm{b}}$ & $8.09^{c}$ & $134.30^{\mathrm{a}}$ & $185.39^{d}$ & $0.15^{\mathrm{c}}$ & $7.48^{\mathrm{e}}$ & $2.34^{\mathrm{b}}$ & $9.82^{\mathrm{d}}$ \\
\hline BGH 4733-55 & $61.03^{\mathrm{b}}$ & $20.52^{\mathrm{d}}$ & $6.21^{\mathrm{d}}$ & $0.70^{\mathrm{c}}$ & $11.65^{\mathrm{b}}$ & $7.84^{\mathrm{c}}$ & $103.66^{\mathrm{a}}$ & $158.30^{\mathrm{d}}$ & $0.18^{\mathrm{c}}$ & $4.88^{\mathrm{i}}$ & $1.17^{\mathrm{h}}$ & $6.05^{\mathrm{j}}$ \\
\hline BGH 4733-56 & $41.35^{\mathrm{d}}$ & $10.66^{\mathrm{f}}$ & $1.86^{\mathrm{e}}$ & $0.33^{\mathrm{d}}$ & $20.92^{\mathrm{a}}$ & $12.90^{\mathrm{a}}$ & $105.47^{\mathrm{a}}$ & $595.84^{\mathrm{a}}$ & $0.11^{\mathrm{c}}$ & $6.97^{\mathrm{f}}$ & $1.70^{\mathrm{f}}$ & $8.67^{\mathrm{f}}$ \\
\hline BGH 4744-57 & $42.81^{\mathrm{d}}$ & $26.27^{\mathrm{c}}$ & $7.47^{\mathrm{d}}$ & $0.85^{\mathrm{b}}$ & $11.80^{\mathrm{b}}$ & $7.47^{\mathrm{c}}$ & $91.89^{\mathrm{b}}$ & $188.45^{\mathrm{d}}$ & $0.23^{\mathrm{b}}$ & $0.00^{\mathrm{q}}$ & $0.02^{\mathrm{m}}$ & $0.03^{\mathrm{s}}$ \\
\hline BGH 4750-59 & $26.69^{\mathrm{e}}$ & $14.03^{\mathrm{e}}$ & $2.46^{\mathrm{e}}$ & $0.42^{\mathrm{d}}$ & $17.43^{\mathrm{a}}$ & $9.31^{\mathrm{b}}$ & $115.96^{\mathrm{a}}$ & $315.24^{\mathrm{b}}$ & $0.17^{\mathrm{c}}$ & $6.84^{\mathrm{f}}$ & $2.16^{\mathrm{c}}$ & $9.00^{\mathrm{e}}$ \\
\hline BGH 4756-67 & $36.23^{\mathrm{d}}$ & $15.07^{\mathrm{e}}$ & $2.90^{\mathrm{e}}$ & $0.38^{d}$ & $13.78^{\mathrm{b}}$ & $7.05^{\mathrm{c}}$ & $127.01^{\mathrm{a}}$ & $99.61^{\mathrm{e}}$ & $0.18^{\mathrm{c}}$ & $6.72^{\mathrm{f}}$ & $1.58^{\mathrm{f}}$ & $8.30^{\mathrm{f}}$ \\
\hline BGH 4756-70 & $23.09^{\mathrm{e}}$ & $12.25^{\mathrm{f}}$ & $1.34^{\mathrm{e}}$ & $0.17^{\mathrm{d}}$ & $13.26^{\mathrm{b}}$ & $9.08^{\mathrm{b}}$ & $135.22^{\mathrm{a}}$ & $72.32^{\mathrm{e}}$ & $0.19^{c}$ & $6.28^{\mathrm{g}}$ & $1.11^{\mathrm{h}}$ & $7.39^{\mathrm{h}}$ \\
\hline BGH 4756-71 & $35.85^{\mathrm{d}}$ & $15.99^{\mathrm{e}}$ & $4.21^{\mathrm{d}}$ & $0.46^{\mathrm{c}}$ & $10.85^{\mathrm{b}}$ & $7.86^{\mathrm{c}}$ & $108.25^{\mathrm{a}}$ & $171.16^{\mathrm{d}}$ & $0.23^{\mathrm{b}}$ & $3.86^{\mathrm{k}}$ & $0.84^{j}$ & $4.70^{1}$ \\
\hline BGH 5012-72 & $25.66^{\mathrm{e}}$ & $10.37^{\mathrm{f}}$ & $1.26^{\mathrm{e}}$ & $0.22^{\mathrm{d}}$ & $17.64^{\mathrm{a}}$ & 9. & $97.12^{\mathrm{a}}$ & $49.28^{\mathrm{e}}$ & $0.14^{\mathrm{c}}$ & $8.30^{\mathrm{d}}$ & $1.23^{\mathrm{h}}$ & $9.53^{\mathrm{d}}$ \\
\hline BGH 5012-76 & $38.75^{\mathrm{d}}$ & $58^{\mathrm{f}}$ & & & $14.42^{\mathrm{a}}$ & & 117 & & $5^{\mathrm{c}}$ & $8^{\mathrm{f}}$ & 1. & $7.93^{\mathrm{g}}$ \\
\hline BGH $6009-78$ & $38.31^{\mathrm{d}}$ & $42.89^{\mathrm{a}}$ & & $1.38^{\mathrm{a}}$ & $7.05^{b}$ & & & & & & & $0.00^{\mathrm{s}}$ \\
\hline BGH 6228-79 & $47.01^{\mathrm{c}}$ & $12.86^{\mathrm{e}}$ & $3.02^{\mathrm{e}}$ & $0.52^{\mathrm{c}}$ & $17.04^{\mathrm{a}}$ & $7.99^{\mathrm{c}}$ & $138.85^{\mathrm{a}}$ & $109.16^{\mathrm{e}}$ & $0.16^{\mathrm{c}}$ & $9.42^{\mathrm{b}}$ & $1.96^{\mathrm{d}}$ & $11.38^{\mathrm{b}}$ \\
\hline BGH 6228-82 & $37.07^{\mathrm{d}}$ & $24.26^{\mathrm{c}}$ & $5.90^{\mathrm{d}}$ & $0.65^{\mathrm{c}}$ & $11.99^{\mathrm{b}}$ & $7.80^{\mathrm{c}}$ & $110.09^{\mathrm{a}}$ & $328.74^{b}$ & $0.23^{\mathrm{b}}$ & $7.76^{\mathrm{e}}$ & $1.60^{\mathrm{f}}$ & $9.35^{\mathrm{d}}$ \\
\hline BGH 6233-83 & $61.53^{\mathrm{b}}$ & $21.16^{\mathrm{d}}$ & $8.50^{\mathrm{c}}$ & $0.86^{\mathrm{b}}$ & $10.68^{b}$ & $7.20^{\mathrm{c}}$ & $76.54^{b}$ & $113.11^{\mathrm{e}}$ & $0.22^{\mathrm{b}}$ & $0.64^{\mathrm{p}}$ & $0.08^{\mathrm{m}}$ & $0.72^{\mathrm{r}}$ \\
\hline BGH 6233-84 & $50.31^{\mathrm{c}}$ & $37.38^{\mathrm{b}}$ & $13.28^{\mathrm{b}}$ & $1.05^{\mathrm{b}}$ & $8.27^{\mathrm{b}}$ & $7.04^{\mathrm{c}}$ & $116.07^{\mathrm{a}}$ & $154.64^{\mathrm{d}}$ & $0.24^{\mathrm{b}}$ & $3.42^{1}$ & $0.66^{\mathrm{k}}$ & $4.08^{\mathrm{m}}$ \\
\hline BGH 6233-85 & $56.94^{\mathrm{b}}$ & $30.55^{\mathrm{c}}$ & $12.40^{\mathrm{b}}$ & $0.95^{\mathrm{b}}$ & $8.06^{\mathrm{b}}$ & $5.93^{c}$ & $101.31^{\mathrm{a}}$ & $214.38^{d}$ & $0.30^{\mathrm{a}}$ & $0.00^{\mathrm{q}}$ & $0.00^{\mathrm{m}}$ & $0.00^{\mathrm{s}}$ \\
\hline BGH 6239-86 & $62.19^{\mathrm{b}}$ & $22.73^{\mathrm{d}}$ & $7.65^{d}$ & $0.90^{\mathrm{b}}$ & $13.02^{\mathrm{b}}$ & $5.97^{\mathrm{c}}$ & $70.60^{\mathrm{b}}$ & $137.93^{\mathrm{d}}$ & $0.17^{\mathrm{c}}$ & $4.37^{\mathrm{j}}$ & $1.44^{\mathrm{g}}$ & $5.81^{\mathrm{j}}$ \\
\hline BGH 6344-87 & $23.74^{\mathrm{e}}$ & $14.89^{\mathrm{e}}$ & $2.40^{\mathrm{e}}$ & $0.45^{\mathrm{c}}$ & $19.13^{\mathrm{a}}$ & $8.69^{b}$ & $112.90^{\mathrm{a}}$ & $44.50^{\mathrm{e}}$ & $0.20^{\mathrm{c}}$ & $2.18^{\mathrm{n}}$ & $0.49^{1}$ & $2.67^{\circ}$ \\
\hline BGH 6369-90 & $32.78^{\mathrm{d}}$ & $32.95^{\mathrm{b}}$ & $11.00^{\mathrm{c}}$ & $0.93^{\mathrm{b}}$ & $9.29^{b}$ & $6.20^{\mathrm{c}}$ & $86.81^{\mathrm{b}}$ & $97.25^{\mathrm{e}}$ & $0.29^{\mathrm{a}}$ & $2.06^{\mathrm{n}}$ & $0.79^{j}$ & $2.85^{\circ}$ \\
\hline BGH 6371-93 & $76.22^{\mathrm{a}}$ & $23.69^{c}$ & $9.38^{\mathrm{c}}$ & $1.33^{\mathrm{a}}$ & $14.52^{\mathrm{a}}$ & $9.18^{b}$ & $51.38^{\mathrm{c}}$ & $152.53^{\mathrm{d}}$ & $0.20^{\mathrm{c}}$ & $0.24^{\mathrm{q}}$ & $0.46^{1}$ & $0.70^{r}$ \\
\hline BGH 6371-94 & $57.57^{\mathrm{b}}$ & $8.69^{f}$ & $2.25^{\mathrm{e}}$ & $0.38^{\mathrm{d}}$ & $17.46^{\mathrm{a}}$ & $8.47^{\mathrm{c}}$ & $38.76^{\mathrm{c}}$ & $286.36^{\mathrm{c}}$ & $0.12^{\mathrm{c}}$ & $2.95^{\mathrm{m}}$ & $0.99^{i}$ & $3.94^{\mathrm{m}}$ \\
\hline BGH 6371-95 & $21.67^{\mathrm{e}}$ & $31.83^{b}$ & $6.90^{\mathrm{d}}$ & $0.65^{\mathrm{c}}$ & $9.56^{\mathrm{b}}$ & $7.80^{\mathrm{c}}$ & $89.41^{\mathrm{b}}$ & $128.47^{\mathrm{d}}$ & $0.24^{\mathrm{b}}$ & $7.58^{\mathrm{e}}$ & $1.88^{\mathrm{d}}$ & $9.46^{\mathrm{d}}$ \\
\hline BGH 6378-98 & $48.74^{\mathrm{c}}$ & $25.89^{\mathrm{c}}$ & $10.58^{\mathrm{c}}$ & $1.03^{\mathrm{b}}$ & $9.37^{\mathrm{b}}$ & $7.17^{\mathrm{c}}$ & $74.91^{\mathrm{b}}$ & $142.16^{\mathrm{d}}$ & $0.29^{\mathrm{a}}$ & $0.00^{\mathrm{q}}$ & $0.00^{\mathrm{m}}$ & $0.00^{\mathrm{s}}$ \\
\hline BGH $6387-100$ & $33.19^{\mathrm{d}}$ & $28.77^{\mathrm{c}}$ & $7.59^{d}$ & $0.83^{b}$ & $10.91^{\mathrm{b}}$ & $8.30^{\mathrm{c}}$ & $96.55^{\mathrm{a}}$ & $81.36^{\mathrm{e}}$ & $0.22^{\mathrm{b}}$ & $4.42^{\mathrm{j}}$ & $0.79^{j}$ & $5.21^{\mathrm{k}}$ \\
\hline BGH $6515-101$ & $14.15^{\mathrm{e}}$ & $11.70^{\mathrm{f}}$ & $1.02^{\mathrm{e}}$ & $0.17^{\mathrm{d}}$ & $16.91^{\mathrm{a}}$ & $8.04^{\mathrm{c}}$ & $107.57^{\mathrm{a}}$ & $108.72^{\mathrm{e}}$ & $0.17^{\mathrm{c}}$ & $8.81^{\mathrm{c}}$ & $1.85^{\mathrm{d}}$ & $10.66^{\mathrm{c}}$ \\
\hline
\end{tabular}

For abbreviations, see legend to Table 2. *Significant at $5 \%$ by the Scott-Knott test. Means followed by the same letter in each columm belong to a homogeneous group at the $5 \%$ probability (Scott-Knott, 1974).

clustered into A together with BGH 4289-44 and BGH 4289-45 with levels above $10.87^{\circ}$ Brix. Next, $24.90 \%$ of the accessions were clustered into group B because of their intermediate levels between 8.59 and $9.69^{\circ}$ Brix. Finally, $69.39 \%$ of the accessions were clustered into group $\mathrm{C}$ because their totals were lower than $8.49^{\circ}$ Brix (Table 3 ).

Vitamin C contents between 38.75 and $138.84 \mathrm{mg} / 100 \mathrm{~g}$ fresh pulp allowed for clustering $61 \%$ of the accessions into group $\mathrm{A}$, in which all of them have vitamin $\mathrm{C}$ concentrations 
higher than $95 \mathrm{mg}$. The averages of BGH 6228-79, BGH 4756-70 and BGH 4733-54 are all higher than $130 \mathrm{mg} / 100 \mathrm{~g}$ fresh pulp (Table 3).

Color intensity ranged from 30.35 to 595.84 ASTA units, and some of these values are higher than the estimates reported by Gomez et al. (1998), who investigated commercial varieties of C. chinensis under greenhouse cultivation. These authors found values between 190 and 360 units under field conditions, where their values ranged between 181 and 335 units. These accessions were clustered into five groups in which A had only the BGH 4733-56 because of its intensive red color followed by cluster B with BGH 1747-26, BGH 6228-82, and BGH 4750-59. In summary, there is genetic variability for fruit color intensity, which depends on the pigment type, although red and orange have the highest magnitudes.

Total capsaicin ranges from trace levels to $13.62 \mathrm{mg} / \mathrm{g}$ dry biomass, and these levels are similar to the data from Gibbs and O'Garro (2004), who reported estimates between 0.25 and 16.55 $\mathrm{mg} / \mathrm{g}$ dry biomass. Total capsaicin showed the highest genetic diversity $(\mathrm{P}<0.05)$, which accounted for the distribution of the accessions among 18 clusters. Accessions with the highest content of capsaicin, such as BGH 1716-17, were clustered into group A, BGH 6228-79 into B, BGH 6515101 into C, BGH 5012-72 into D, and BGH 6228-82 into E. Other accessions had intermediate estimates, except those with sweet fruit, such as BGH 1716-14, BGH 1716-16, BGH 4201-32, BGH 4223-39, BGH 4731-53, BGH 6009-78, BGH 6233-85, and BGH 6378-98 (Table 3).

The genetic variability of capsaicin ranges from 0.00 to $10.32 \mathrm{mg} / \mathrm{g}$ dry biomass, and 17 clusters were formed $(\mathrm{P}<0.05)$. The dihydrocapsaicin, otherwise, ranges from 0.00 to 3.42 $\mathrm{mg}$, but the accessions were clustered into 13 groups. All of them have higher levels of capsaicin than dihydrocapsaicin, except BGH 6371-93 (Table 3). According to Bosland (1993), the different combinations of capsaicinoids induce individual characteristics of fruit pungency, although both capsaicinoids account for the same taste of heat.

The multi-categorical descriptors (IPGRI, 1995) showed genetic variability (Table 1). The growth of most accessions (53.06\%) is classified as compact, and they are followed by those with erect growth (44.89\%). Just BGH 1716-14 grows prostrated on the ground. Stems with scarce pubescence are found in $61.22 \%$ of the accessions followed by those with intermediate $(28.57 \%)$, smooth $(6.12 \%)$ and abundant hairs $(4.08 \%)$. In terms of stems, green is the predominant color $(81.63 \%)$, but in the other accessions they are purple. The color of the corolla is whitish in $95.91 \%$ of the accessions and white-greenish in the others. In immature fruit, the predominant color is green $(87.75 \%)$, followed by yellow $(8.16 \%)$ and purple $(4.08 \%)$. Mature fruit are red (61.22\%), orange $(32.65 \%)$ and yellow $(6.12 \%)$.

In terms of shape (Table 1), there is a high percentage of accessions with longitudinal fruit (47.0\%) followed by those with the conical fruit (25.0\%). The other accessions have campanular $(10.0 \%)$, bell $(12.0 \%)$, triangular $(4.0 \%)$, and rounded fruit $(2 \%)$. Most of the accessions have pointed fruit tips (83.67\%), but others have no tips (4.08\%) or sunken tips (12.25\%).

Thirteen RAPD primers (41.0\%) in 32 are polymorphic. Forty-four bands (15.4\%) are polymorphic for the identified loci in 286 amplifications. Similar results were reported by Paran et al. (1998), who studied the genetic diversity of C. annuum and found $20.0 \%$, and Rodriguez et al. (1999), who studied numerous species in the genus Capsicum, also found the same $20 \%$.

The variance and covariance matrices detected severe multicollinearity. Therefore, the contents of capsaicin and dihydrocapsaicin were eliminated before the multivariate analysis because of their high correlations with total capsaicin, and their substantial contribution to multicollinearity. Furthermore, the generalized Mahalanobis distance evaluated the redundancy in the physicochemi- 
cal traits from which only the total dry biomass was eliminated because of the autovalue in the last autovector detected by the canonical variables. In terms of the relative contribution of physicochemical traits, total capsaicin accounts for $88.88 \%$ of the variation followed by fruit width (2.65\%), fruit length $(2.10 \%)$, fruit dry matter (1.85\%), extractable fruit color $(1.76 \%)$, vitamin C $(1.14 \%)$, soluble solids $(0.87 \%)$, pulp thickness $(0.73)$, and percentage of fruit dry matter $(0.22 \%)$. These results accounted for the variation in total capsaicin, which contributed to the genetic divergence.

Tocher's method, cited by Rao (1952), using Mahalanobis' genetic distance (1936), clustered all the accessions into nine groups (Table 4). The cluster I consists of all the accessions with sweet fruit, including BGH 1716-14, BGH 1716-16, BGH 4201-32, BGH 422339, BGH 4731-53, BGH 4744-57, BGH 6233-85, and BGH 6378-98, except BGH 6009-78, which is clustered into IX. All accessions in the clusters II, III, V, VI, VII, and VII contain concentrations of total capsaicin from average to high levels, but the most pungent accessions such as BGH 1716-17 is clustered into VI, BGH 4733-54 and BGH 6228-79 are clustered into $\mathrm{V}$, and BGH 6515-101 is clustered into group III. Otherwise, BGH 1747-26, BGH 6344-87 and BGH 6369-90 are clustered into group IV because of the total capsaicin from low to aver-

Table 4. Clustering of 49 accessions of Capsicum chinensis from the BGH-UFV by Tocher's method, according to physicochemical characteristics of fruit, multi-categorical traits, RAPD markers, and combined analysis.

\begin{tabular}{|c|c|c|}
\hline Characteristics & Cluster & Accessions \\
\hline \multirow[t]{9}{*}{ Physicochemical } & I & $7^{*}, 9,11,14,16,19,32,39,53,57,83,85,93,98$ \\
\hline & II & $5,6,18,22,27,30,34,40,45,46,51,55,7184,86,100$ \\
\hline & III & $23,44,59,67,70,72,76,82,95,101$ \\
\hline & IV & $26,87,90$ \\
\hline & $\mathrm{V}$ & 54,79 \\
\hline & VI & 17 \\
\hline & VII & 56 \\
\hline & VIII & 94 \\
\hline & IX & 78 \\
\hline \multirow[t]{4}{*}{ Multi-categorical } & I & $\begin{array}{l}6,7,9,11,14,16,17,18,19,23,26,27,30,32,39,40,44,45,51,53,54,55, \\
56,57,59,67,70,71,72,76,78,79,82,83,84,85,86,90,93,94,95,98\end{array}$ \\
\hline & II & $5,22,34,46,101$ \\
\hline & III & 100 \\
\hline & IV & 87 \\
\hline \multirow[t]{8}{*}{ RAPD } & I & $\begin{array}{l}5,6,7,14,16,18,19,22,23,26,27,30,32,34,39,40,45,46,51,53 \\
54,55,56,57,67,70,71,76,78,79,82,83,84,85,87,95,98,101\end{array}$ \\
\hline & II & 93,94 \\
\hline & III & $9,11,59$ \\
\hline & IV & 86,100 \\
\hline & V & 72 \\
\hline & VI & 90 \\
\hline & VII & 44 \\
\hline & VIII & 17 \\
\hline \multirow[t]{14}{*}{ Combined analysis } & I & $5,6,14,16,17,18,19,32,45,51,55,67,70,71,78,82,83,85$ \\
\hline & II & 93,94 \\
\hline & III & $7,9,11,84$ \\
\hline & IV & $26,27,30,39$ \\
\hline & $\mathrm{V}$ & $22,56,59$ \\
\hline & VI & 40,54 \\
\hline & VII & $46,72,76,79$ \\
\hline & VIII & 90,98 \\
\hline & IX & $34,87,95,101$ \\
\hline & $\mathrm{X}$ & 57,100 \\
\hline & XI & 23 \\
\hline & XII & 86 \\
\hline & XIII & 53 \\
\hline & XIV & 44 \\
\hline
\end{tabular}

\footnotetext{
*In this table, the accessions are represented by their two final numbers.
} 
age levels, and BGH 6371-94 is clustered alone into group VIII.

Tocher's method based on the matrices of Cole-Rodgers et al. (1997) for dissimilarities formed four clusters, and the Nei and Li (1979) matrices formed eight (Table 4). Both methods were poor in discriminating the accessions, and their results were found in disagreement with the Mahalanobis distance. This disagreement is verified by the absence of significant differences detected by the Mantel test when the pairs of distance matrices are compared. Therefore, the combined analysis was used to form 14 clusters by Tocher's method after the sum of the standardized distance with $36.7 \%$ of the accessions (Table 4). Significant correlations with the Mahalanobis $(r=0.55)$, Cole-Rodgers $(r=0.59)$ and Nei and Li $(r=0.60)$ distance matrices were detected by the Mantel test (10,000 simulations) to verify the association between these matrices and the individual analyses. These results showed the equitable contribution of the different traits to genetic diversity without the bias of the Gower algorithm (1971), because of moderate contribution in the combined matrices, which depends on the number of traits.

Sweet fruit (BGH 1716-14, BGH 1716-16, BGH 4201-32, and BGH 6233-85) and less pungent accessions such as BGH 1716-19 and BGH 6233-83 were clustered according to physicochemical characteristics, and they maintained here the same constitution because of their great genetic similarities. Otherwise, the accession with sweet fruit (BGH 6009-78), which was alone in cluster IX, was then grouped into cluster I by the combined analysis.

In terms of origin, the use of geographic sites to discriminate the accessions revealed inconsistency. BGH 6369-90, BGH 6371-93, BGH 6371-94, and BGH 6371-95 collected in Vila Nova-Anaman, AM, were distributed in the cluster II (BGH 6371-93 and BGH 6371-94), VIII (BGH 6369-90 and BGH 6378-98) and IX (BGH 6371-95). BGH 6371-95, together with the accessions collected in Tefé, AM (BGH 4213-34), Dourados, MS (BGH 6344-87) and Viçosa, MG (BGH 6515-101) consolidated the cluster IX. The accessions collected in Pindaré-Mirim, AM, BGH 1716-14, BGH 1716-16, BGH 1716-17, BGH 1716-18, and BGH 1716-19 were clustered into I, and BGH 1716-9 and BGH 1716-11 were clustered into III together with BGH1694-7 and BGH 6233-84, collected in Cuiabá, MT, and Brasília, DF, respectively. Other accessions from Brasília, DF, were clustered into I (BGH 6228-82, BGH 6233-83 and BGH 6233-85), but the cluster VII had the BGH 6228-79 and XII had the BGH 6239-86, only. The above accessions and clusters showed no relationship between genetic diversity and geographic distance. According to Murty and Arunachalan (1966) and Upadhaly and Murty (1970), both cited by Cruz and Carneiro (2003), genetic drift and plant selection in different environments can have more significant effects on diversity than the geographical location. Nevertheless, genetic contamination during seed multiplication can affect these responses, because cross pollination in the Capsicum genus ranges from 0.5 to $70 \%$ (Cruz and Carneiro, 2003), although the plants in the current study were protected by white cloth to reduce the chances of contamination.

Regardless of the accession origin, clustering is essential for selecting divergent $C$. chinensis genotypes. For example, within cluster I, which accounted for $36.7 \%$ of accessions, it is possible to select accessions such as BGH 1716-17 to participate in breeding programs because of its fruit pungency. Thus, this accession can be intercrossed, for example, with BGH 4223-39 and BGH 6233-84 from other clusters because of their fruit size.

Otherwise, as the analysis of genetic diversity is predictive because it does not consider the gene effects of traits, the crosses can produce progeny with unsatisfactory agronomic responses. Nonetheless, the use of multivariate analyses to identify divergent accessions may be essential for organizing the diallel permitting the evaluation of the types of gene effects and 
the crossing of pairs with better chances of success. Therefore, the most promising pungent accessions to constitute the diallel are BGH 1716-17, BGH 4289-44, BGH 4733-54, BGH 501272, BGH 6228-79, and BGH 6515-101. Otherwise, the better accessions with sweet fruit are BGH 1716-16, BGH 4201-32, BGH 4223-39, BGH 4731-53, BGH 6233-85, and BGH 637898, of which BGH 1716-16, BGH 4201-32 and BGH 6233-85 were clustered into group I.

The genetic control of fruit pungency, which has been considered as dominant monogenic in most reports (Greenleaf, 1986; Stewart et al, 2007), has been easy to identify after controlled crosses. Therefore, in-depth exploration of this genetic variability can be performed using diallel crosses where the accessions have a different level of pungency. Finally, the formation of clusters to study the genetic control of pungency and sweetness using partial diallel crosses may also be an interesting genetic strategy.

\section{ACKNOWLEDGMENTS}

\section{Research supported by FAPEMIG, CAPES/DAAD and IPEAC/UNIPAR.}

\section{REFERENCES}

Bosland PW (1992). Chiles: a diverse crop. Hort. Techn. 2: 6-10.

Bosland PW (1993). Breeding for quality Capsicum. Capsicum Eggplant Newsl. 12: 25-31.

Buso GSC, Amaral ZPS, Bianchetti LB, Machado FRB, et al. (2003). Genetic variability and phylogenetic analysis of Brazilian species of Capsicum. Capsicum Eggplant Newsl. 22: 1-3.

Carvalho SIC, Bianchetti LB, Bustamante PG and Silva DB (2003). Catálogo de Germoplasma de Pimentas e Pimentões (Capsicum spp) da Embrapa Hortaliças. Embrapa Hortaliças, Brasília.

Casali VWD and Couto FA (1984). Origem botânica de Capsicum. Inf. Agrop. 113: 8-10.

Casali VWD and Strigheta PC (1984). Melhoramento de pimentão e pimenta para fins industriais. Inf. Agrop. 10: 23-25.

Casali VWD, Pádua JG and Braz LT (1984). Melhoramento de pimentão e pimenta. Inf. Agrop. 10: 19-22.

Cole-Rodgers P, Smith DW and Bosland PW (1997). A novel statistical approach to analyze genetic resource evaluations using Capsicum as an example. Crop Sci. 37: 1000-1002.

Cruz CD (2006a). Programa Genes: Estatística Experimental e Matrizes. Editora UFV, Viçosa.

Cruz CD (2006b). Programa Genes: Biometria. Editora UFV, Viçosa.

Cruz CD and Carneiro PCS (2003). Modelos Biométricos Aplicados ao Melhoramento Genético. Editora da UFV, Viçosa.

Fulton TM, Chunwongse J and Tanksley SD (1995). Microprep protocol for extraction of DNA from tomato and herbaceous plants. Plant Mol. Biol. Rep. 13: 207-209.

Gibbs HAA and O'Garro LW (2004). Capsaicin content of West Indies hot pepper cultivars using colorimetric and chromatographic techniques. Hort. Sci. 39: 132-135.

Gomez R, Pardo JE, Navarro F and Varón R (1998). Color differences in paprica pepper varieties (Capsicum annuum L.) cultivated in a greenhouse and in the open air. J. Sci. Food Agric. 77: 268-272.

Gonçalves LSA, Rodrigues R, Amaral Junior AT and Karasawa M (2008). Comparison of multivariate statistical algorithms to cluster tomato heirloom accessions. Genet. Mol. Res. 7: 1289-1297.

Gower JC (1971). A general coefficient of similarity and some of its properties. Biometrics 27: 857-874.

Greenleaf W (1986). Pepper Breeding. In: Breeding Vegetable Crops (Basset MJ, ed.). AVI, Connecticut, 69-134.

Instituto Adolfo Lutz (1985). Normas Analíticas do Instituto Adolfo Lutz; Métodos Químicos e Físicos para Análises de Alimento. Instituto Adolfo Lutz, São Paulo.

IPGRI (International Plant Genetic Resources Institute) (1995). Descriptors for Capsicum (Capsicum spp). International Plant Genetic Resources Institute, Rome.

Kawada T, Watanabe T, Katsura K, Takami H, et al. (1985). Formation and metabolism of pungent principle of Capsicum fruits. XV. Microdetermination of capsaicin by high-performance liquid chromatography with electrochemical detection. J. Chromatogr. 329: 99-105.

Kumar V, Sharma S, Sharma AK and Sharma S (2009). Comparative analysis of diversity base on morpho-agronomic traits and microsatellite markers in common bean. Euphytica 170: 249-262. 
Lannes SD, Finger FL, Schuelter AR and Casali VWD (2007). Growth and quality of Brazilian accessions of Capsicum chinense fruits. Scient. Hortic. 112: 266-270.

Mahalanobis PC (1936). On the generalized distance in statistics. Proc. Natl. Inst. Sci. India 2: 49-55.

Maillard MN, Giampaoli P and Richard HJ (1997). Analysis of eleven capsaicinoids by reversed-phase high performance liquid chromatography. Flav. Fragr. J. 12: 409-413.

Mantel N (1967). The detection of disease clustering and a generalized regression approach. Cancer Res. 27: 209-220.

Moscone EA, Scaldaferro MA, Grabiele M and Cecchini NM, et al. (2007). The evolution of chilli peppers (Capsicum Solanaceae): a cytogenetic perspective. Acta Hort. 745: 137-170.

Nei M and Li WH (1979). Mathematical model for studying genetic variation in terms of restriction endonucleases. Proc. Natl. Acad. Sci. U. S. A. 76: 5269-5273.

Oliveira VR, Casali VWD, Cruz CD and Pereira PR (1999). Avaliação da diversidade genética em pimentão através de análise multivariada. Hortic. Bras. 17: 19-24.

Paran I, Aftergoot E and Shifriss C (1998). Variation in Capsicum annuum revealed by RAPD and AFLP markers. Euphytica 99: 167-173.

Pereira GM (2007). Análise Dialélica e Pungência de Frutos em Capsicum chinense. Doctoral thesis, UFV, Viçosa.

Rao CR (1952). An Advanced Statistical Method in Biometric Research. John Wiley and Sons, New York.

Rêgo ER, Rêgo MM, Cruz CD and Cecon PR (2003). Genetic diversity analysis of peppers: a comparison of discarding variable methods. Crop Breed. Appl. Biotech. 3: 19-26.

Reifschneider FJB (2000). Capsicum: Pimentas e Pimentões no Brasil. Embrapa Comunicação para Transferência de Tecnologia. Embrapa Hortaliças, Brasília.

Rodriguez JM, Berke T, Engle L and Nienhuis J (1999). Variation among and within Capsicum species revealed by RAPD markers. Theor. Appl. Genet. 99: 147-156.

Schuelter AR, Pereira GM, Amaral Junior AT and Casali VWD (2010). Genetic control of agronomically important traits of pepper fruits analyzed by Hayman's partial diallel cross scheme. Genet. Mol. Res. 9: 113-127.

Scott AT and Knott M (1974). A cluster analysis method for grouping means in the analysis of variance. Biometrics 30 : 507-512.

Segatto FB (2007). Avaliação da Qualidade "Pós-Produção" de Pimenta Ornamental (Capsicum annuum) Cultivadas em Vaso. Doctoral thesis, UFV, Viçosa.

Singh D (1981). The relative importance of characters affecting genetic divergence. Ind. J. Gen. Plant Breed. 41: 237-245. Souza RJ and Casali WD (1984). Cultivares de pimentão e pimenta. Inf. Agrop. 113: 15-18.

Stewart C Jr, Mazourek M, Stellari GM, O'Connell M, et al. (2007). Genetic control of pungency in C. chinense via the Pun1 locus. J. Exp. Bot. 58: 979-991.

Stommel JR and Bosland PW (2006). Ornamental Pepper, Capsicum апnиum. In: Flower Breeding and Genetics: Issues, Challenges and Opportunities for the 21st Century (Anderson N, ed.). Springer, Dordrecht, 561-599.

Teixeira R (1996). Diversidade em Capsicum: Análise Molecular, Morfoagronômica e Química. Master's thesis, UFV, Viçosa.

Toquica SP, Rodríguez F, Martinez E, Duque MC, et al. (2003). Molecular characterization by AFLPs of Capsicum germplasm from the Amazon department in Colombia. Gen. Res. Crop Evol. 50: 639-647.

Williams JG, Kubelik AR, Livak KJ, Rafalski JA, et al. (1990). DNA polymorphisms amplified by arbitrary primers are useful as genetic markers. Nucleic Acids Res. 18: 6531-6535. 